\title{
Wet Etched High Aspect Ratio Microstructures on Quartz for MEMS Applications
}

\author{
Jinxing Liang Member (Sakamoto Electric Mfg. Co. Ltd., liang@kyushu.rise.waseda.ac.jp) \\ Fusao Kohsaka Student Member (Waseda University) \\ Takahiro Matsuo Student Member (Waseda University) \\ Toshitsugu Ueda Member (Waseda University, t-ueda@waseda.jp)
}

Keywords : quartz, MEMS, anisotropic etching, high aspect ratio, microcapillary, tilt sensor

\section{Introduction}

$\alpha$-quartz draws great attention for its piezoelectricity, excellent mechanical characteristics and electrical insulation in comparison with the conventional MEMS material, silicon. The major obstacles limiting the wide use of $\alpha$-quartz are its complex anisotropic etching profile and the lack of fabrication technologies. This research focused on wet etching of z-cut quartz wafers for high aspect ratio trench and through-hole, which are desired in MEMS fabrication.

\section{Experiment}

Experimental mask patterns were set up with initial widths of 5, $10,20,50,100$ and $200 \mu \mathrm{m}$, the large width $200 \mu \mathrm{m}$ pattern was for observing the whole etching profile and minimum $5[\mu \mathrm{m}]$ pattern was for discussing the possible high aspect ratio microstructure [aspect ratio=etch depth/(undercut + initial width)]. The intermediate initial widths patterns were designed to observe the effect of initial width an etching time on the aspect ratio. A 100 $\mu \mathrm{m} \mathrm{Z}$ cut wafer was used in this research, and $\mathrm{Au} / \mathrm{Cr}$ thin film was deposited as the etch mask. Saturated ammonium bifluoride solution at 87 degrees $\mathrm{C}$ was used as the quartz etchant.

\section{Results}

After 40 [min] etching, samples were cut vertical to the etching line and cross sections were observed using the SEM (scanning electron microscope). Etching profile was plotted at a polar interval of $5^{\circ}$. The etch rate at the $\mathrm{Z}$ plane was $110 \mu \mathrm{m} / \mathrm{h}$, which was the same at all polar direction. High aspect ratio structure was obtained at the polar angles from $30^{\circ}$ to $60^{\circ}$. Fig. 1 (a) and (c) show the basic structure of high aspect trench, and the etching aspect ratio dependence on the polar angle after 20, 40, $70 \mathrm{~min}$ etching respectively. The aspect ratio is defined as etch depth to (etching undercut+initial width). An example of experimental micrograph (etched at polar angle $\beta$ of $45^{\circ}$ for $70 \mathrm{~min}$ ) with a etch depth $50.7 \mu \mathrm{m}$ and top width $16.4 \mu \mathrm{m}$ is presented in Fig.1 (b).

\section{Conclusion and Applications}

High aspect ratio microstructure ( $>3$ ) which was considered impossible by wet etching technology was achieved. This result suggests that anisotropic etching of $Z$ cut $\alpha$-quartz can be applied to microcapillary electrophoresis, where high aspect ratio microchannel is desired for improving detecting sensitivity. Trench at polar angle of $45^{\circ}$ is demonstrated to be the best choice. We successfully fabricated nearly vertical trench feature at $\beta=45^{\circ}$ with $50.7 \mu \mathrm{m}$ depth and $16.4 \mu \mathrm{m}$ width, the aspect ratio of 3.1 .
Double-sided etching showed great interest for the twofold symmetry around the $\mathrm{X}$ axis (Fig. 2). According to this property, two same sensors at $\left(\beta=15^{\circ}\right.$ and $\left.105^{\circ}\right)$ and $\left(\beta=-45^{\circ}\right.$ and $\left.45^{\circ}\right)$ can be arranged at $90^{\circ}$ on one quartz chip, which propose a novel two axis tilt sensor.

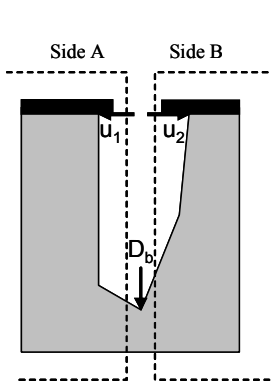

(a)

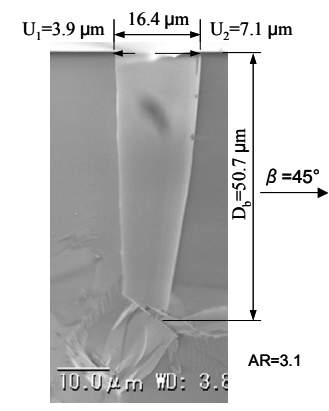

(b)

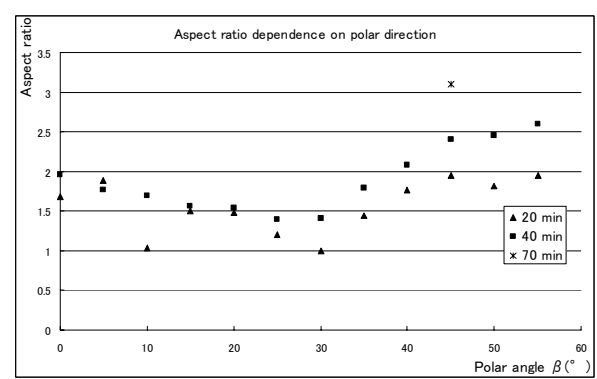

(c)

Fig. 1. Definition of the basic microstructure and aspect ratio: (a) the basic microstructure, $\mathrm{u}_{1}, \mathrm{u}_{2}$, and $\mathrm{D}_{\mathrm{b}}$ represent side etch and bottom etch depth respectively; (b) SEM photograph of 70 min etched trench at $45^{\circ}$; (c) Plotted trench aspect ratio $\left[\mathrm{AR}=\mathrm{D}_{\mathrm{b}} /(\right.$ initial width $\left.+\mathrm{u}_{1}+\mathrm{u}_{2}\right)$ ] dependence on the polar direction based on the $20,40,70$ min etching results

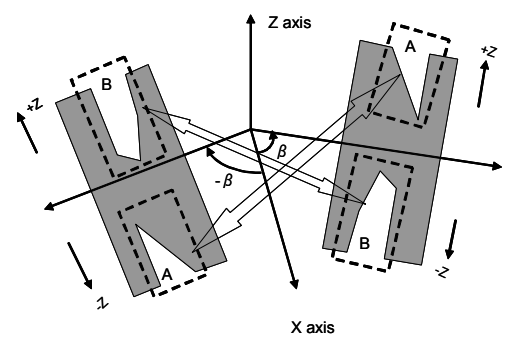

Fig. 2. The twofold symmetry around $\mathrm{X}$ axis 


\title{
Wet Etched High Aspect Ratio Microstructures on Quartz for MEMS Applications
}

\author{
Jinxing Liang* \\ Member \\ Fusao Kohsaka** \\ Student Member \\ Takahiro Matsuo** \\ Student Member \\ Toshitsugu Ueda** \\ Member
}

\begin{abstract}
$\mathrm{Z}$ cut $\alpha$-quartz wafers were etched in saturated ammonium bifluoride solution at 87 degrees $\mathrm{C}$. The side wall profiles were observed using the scanning electron microscopy (SEM) and plotted dependent on the polar direction. This research focused on investigating high aspect ratio trench and through-hole, which were dependent on the polar direction to the crystal axis. Aspect ratio in dependence on polar direction was also plotted and microchannels with aspect ratio $>3$ could be achieved at the polar angle between $30^{\circ}$ to $60^{\circ}$. The possibility of application for microcapillary was discussed, and the trench at $45^{\circ}$ was considered best. Double-sided etching technique was used for manufacturing through-hole structures. Through-hole at $0^{\circ}$ was demonstrated effective for fabrication of capacitive MEMS tilt sensor. Through-holes at $15^{\circ}$ and $105^{\circ}$ were proposed for fabrication of $90^{\circ}$-arranged two axis capactive tilt sensor, taking advantage of the twofold symmetry property around $\mathrm{X}$ axis and threefold symmetry property around $\mathrm{Z}$ axis.
\end{abstract}

Keywords : quartz, MEMS, anisotropic etching, high aspect ratio, microcapillary, tilt sensor

\section{Introduction}

Quartz draws great attention for its piezoelectricity, excellent mechanical characteristics and electrical insulation in comparison with the conventional MEMS material, silicon. Further, it is also well known to be the most appropriate material for bioMEMS, especially for microcapillaries because it is UV transparent and chemically inert. The major obstacles limiting the wide use of quartz wafers are its complex anisotropic etching profile and the lack of fabrication technologies.

The precise $3 \mathrm{D}$ microstructures with a high aspect ratio are needed in many advance applications ${ }^{(1)}$. For example, high aspect ratio microchannel would provide high sensitivity in the microcapillary electrophoresis ( $\mu$-CE) when using UV absorption detection, because absorbance is proportional to the optical path length ${ }^{(2)}$. Current $\mathrm{CE}$ microdevices are mostly fabricated by isotropic etching fused silica or glass, which suffer from the shallow channel depths, because the resulting channels are twice as wide as they are deep. For solving this problem, several groups have made great efforts in recent decades. Ujiie et al. ${ }^{(3)}$ sucessfully fabricated vertical trench features with $50 \mu \mathrm{m}$ depth and $20 \mu \mathrm{m}$ width, the aspect ratio of 2.5 , using dry etching technology. But dry etching method is an expensive process and special equipment is needed. Harrison et al. ${ }^{(4)}$ reported a planar device design with an increased sensitivity, in which the measuring beam was launched through a U-cell, increasing the path length by allowing the beam to pass in a longitudinal direction along the flow channel. Unfortunately, the insertion of optical fibers into etched channels within the device made it difficult to manufacture. Further,

* Sakamoto Electric Mfg. Co. Ltd

3-27-55 Wajiro, Higashi-ku, Fukuoka 811-0202

** Waseda University

The Graduate School of Information, Production and Systems

2-7 Hibikino, Wakamatsu-ku, Kitakyushu, Fukuoka 808-0135 capacitance based sensor is commonly used for acceleration, tilt detection. In this case, high aspect ratio through-hole derives big capacitance and high sensitivity.

Ueda et al. ${ }^{(5)}$ systematically studied the etching anisotropy of quartz by using 21 kinds of cut angles twenty years ago, and after that Cheng et al. ${ }^{(6)}$ reported characterization results of anisotropic etching properties using a spherical specimen. But the undoubted fact is that the actual application of quartz for MEMS was very few in comparison with Si. Z cut quartz wafers show the most potentials to fabricate microsensors and microactuators due to the high etch rate at the $\mathrm{Z}$ plane. Hedlund et al. ${ }^{(7)}$ anisotropically etched $\mathrm{z}$ cut quartz using the mixture solution of $\mathrm{HF}$ and $\mathrm{NH}_{4} \mathrm{~F}$ at different concentrations, temperatures and gave the etching profiles. But the etching rates of side wall planes were not provided, which should be important in designing microdevices. This research experimentally studied wet-etching profile and etching rates of appeared etching planes using traditional quartz wafer etchant, saturated ammonium bifluoride solution at 87 degrees C. Furthermore, we focused on discussing the possible high aspect ratio microstructures and introducing the proposed and prospective MEMS applications, such as MEMS tilt sensor, microcapillary.

\section{Experimental}

2.1 Mask Pattern Design Experimental mask patterns were set up with initial widths of 5, 10, 20, 50, 100 and $200 \mu \mathrm{m}$ (Fig. 1). Large width $200 \mu \mathrm{m}$ pattern was for observing the whole etching profile and minimum $5 \mu \mathrm{m}$ was for discussing the possible high aspect ratio microstructre [Aspect ratio = etch depth/(undercut + initial width)]. The intermediate spaces were design to observe the procedure of disappering of etching plane. Because $\alpha$-quartz is threefold symmetry, we just studied the polar angle from $0^{\circ}$ to $120^{\circ}$ with an interval of $5^{\circ}$ at the beginning of $+\mathrm{x}$ 


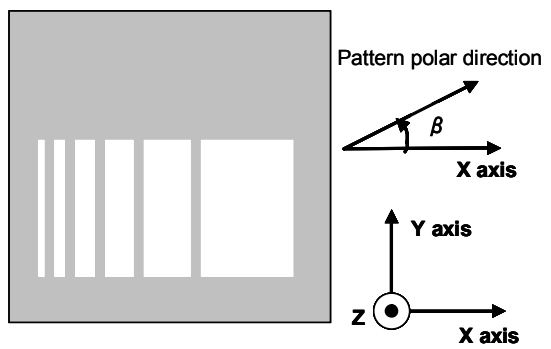

Fig. 1. Designed etch mask with initial spaces of 5, 10, 20, 50, 100 and $200 \mu \mathrm{m}$ (from left to right) and definition of the pattern polar direction $\beta$ starting from $+\mathrm{X}$ axis

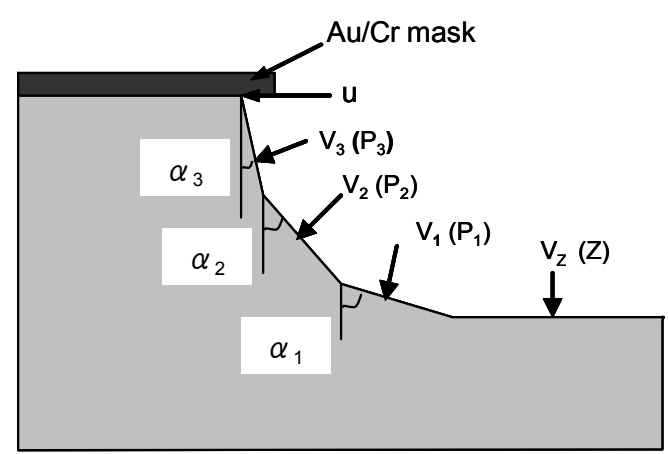

Fig. 2. Definition of the sidewall profile with plane $P_{1}$, $\mathrm{P}_{2}, \mathrm{P}_{3}$ and $\mathrm{Z}$, and the angle $\alpha_{1}, \alpha_{2}, \alpha_{3}$ and etching rate $\mathrm{V}_{1}$, $\mathrm{V}_{2}, \mathrm{~V}_{3}, \mathrm{~V}_{\mathrm{Z}}$ and $\mathrm{u}$

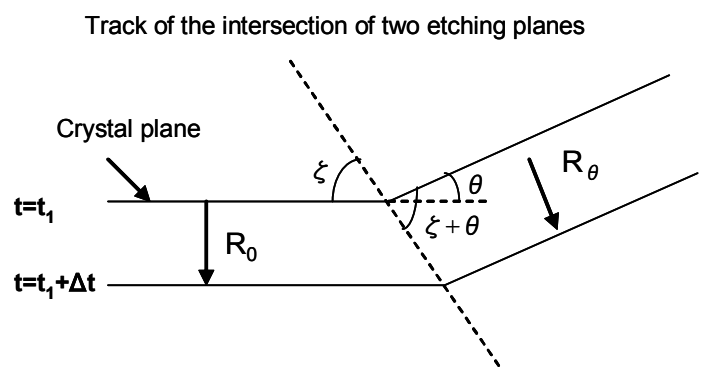

Fig. 3. Etching planes and the track of their intersection

axis.

2.2 Quartz Etching Process A $100 \mu \mathrm{m} \mathrm{Z}$ cut quartz was used in this research. The etching process was as follow. (1) Washing quartz wafers in piraha solution $\left(\mathrm{H}_{2} \mathrm{SO}_{4}: \mathrm{H}_{2} \mathrm{O}_{2}=3: 1\right)$ at 110 degrees C; (2) Sputtering $\mathrm{Cr}(50 \mathrm{~nm})$ and $\mathrm{Au}$ (180nm) mask on the quartz wafer; (3) Patterning the $\mathrm{Cr}$ and $\mathrm{Au}$ thin films with the designed photoresist mask; (4) Etching quartz wafer in saturated ammomium bifluoride solution at 87 degrees C. In this research, a stirrer was not used and no chemicals or water were supplemented.

\subsection{Analysis Method}

2.3.1 Definition of Etching Profile After substrate etching, samples were cut vertically to the etching line and cross sections were observed using scanning electron microscopy (SEM). Etching profile was defined in Fig. 2.

\subsubsection{Track of the Intersection of Two Etching Planes}

The appeared etching planes would advance parallelly and the
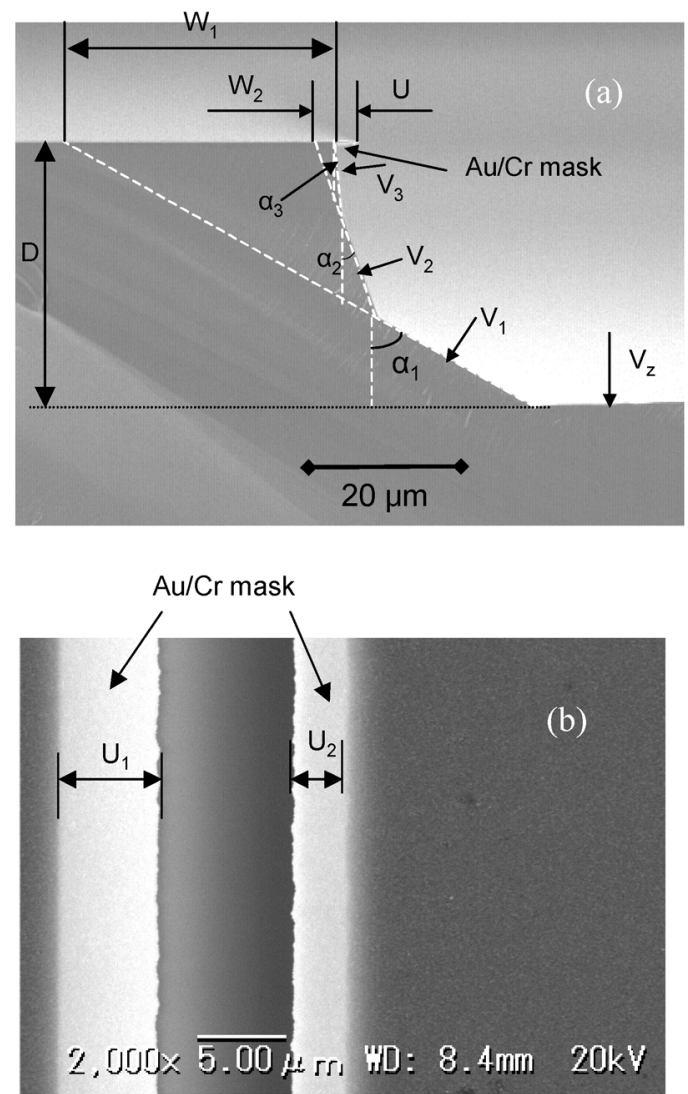

Fig. 4. Examples of SEM picture for measuring sidewall profiles, etching rates (a); etching undercut (b)

intersection of the etching planes is decided by the following formula $(1)^{(8)}$.(Fig. 3)

$$
R_{\theta} \sin \zeta=R_{0} \sin (\zeta+\theta)
$$

\subsubsection{Observing and Calculating the Etching Profiles}

Fig. 4(a) shows an example of the SEM picture for measuring the etching profile. Fig. 4(b) gives an example picture for measuring undercut. The undercut was clearly observed at the SEM photograph (Fig. 4(b)), the dark and bright part represent films with and without quartz, respectively.

Based on the Fig. 3, the etching rates of side wall $\mathrm{P}_{1}, \mathrm{P}_{2}, \mathrm{P}_{3}$ and the $\mathrm{Z}$ plane can be calculated by the following formula (2), (3), (4) and (5), respectively.

$$
\begin{aligned}
& V_{1}=\frac{\left(W_{1}+U\right) \cos \alpha_{1}}{t} \\
& V_{2}=\frac{\left(W_{2}+U\right) \cos \alpha_{2}}{t} \\
& V_{3}=\frac{U \cos \alpha_{3}}{t} \\
& V_{Z}=\frac{D}{t}
\end{aligned}
$$

$V_{1}, V_{2}, V_{3}$ : etching rate of side wall $P_{1}, P_{2}$ and $P_{3}$ respectively $\mathrm{V}_{\mathrm{Z}}$ : etching rate of $\mathrm{Z}$ plane

$\mathrm{U}$ : etching undercut

$\mathrm{t}$ : etching time

$\mathrm{W}_{1}\left(\mathrm{~W}_{2}\right)$ : distance between the intersection of $\mathrm{P}_{3}\left(\mathrm{P}_{2}\right), \mathrm{Au} / \mathrm{Cr}$ mask 


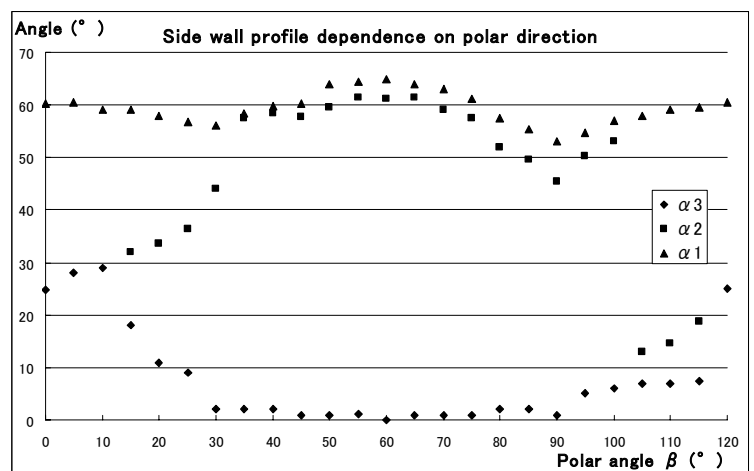

(a)

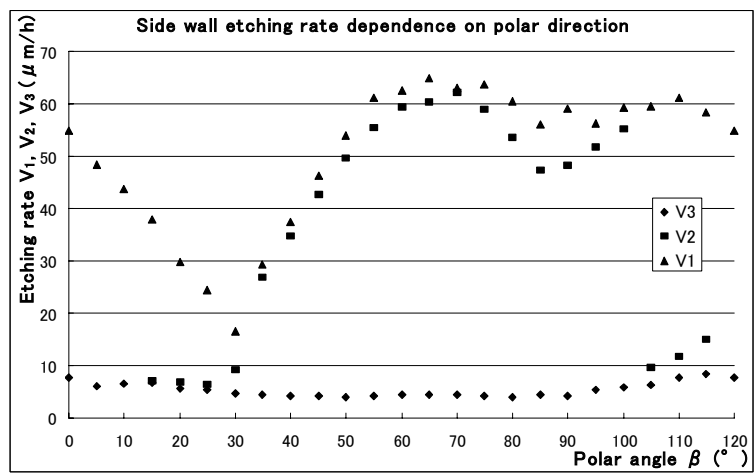

(b)

Fig. 5. Plotted etching profiles dependence on polar angle. Side wall inclination angle (a); Etching rate (b)

Profile dependence on initial width

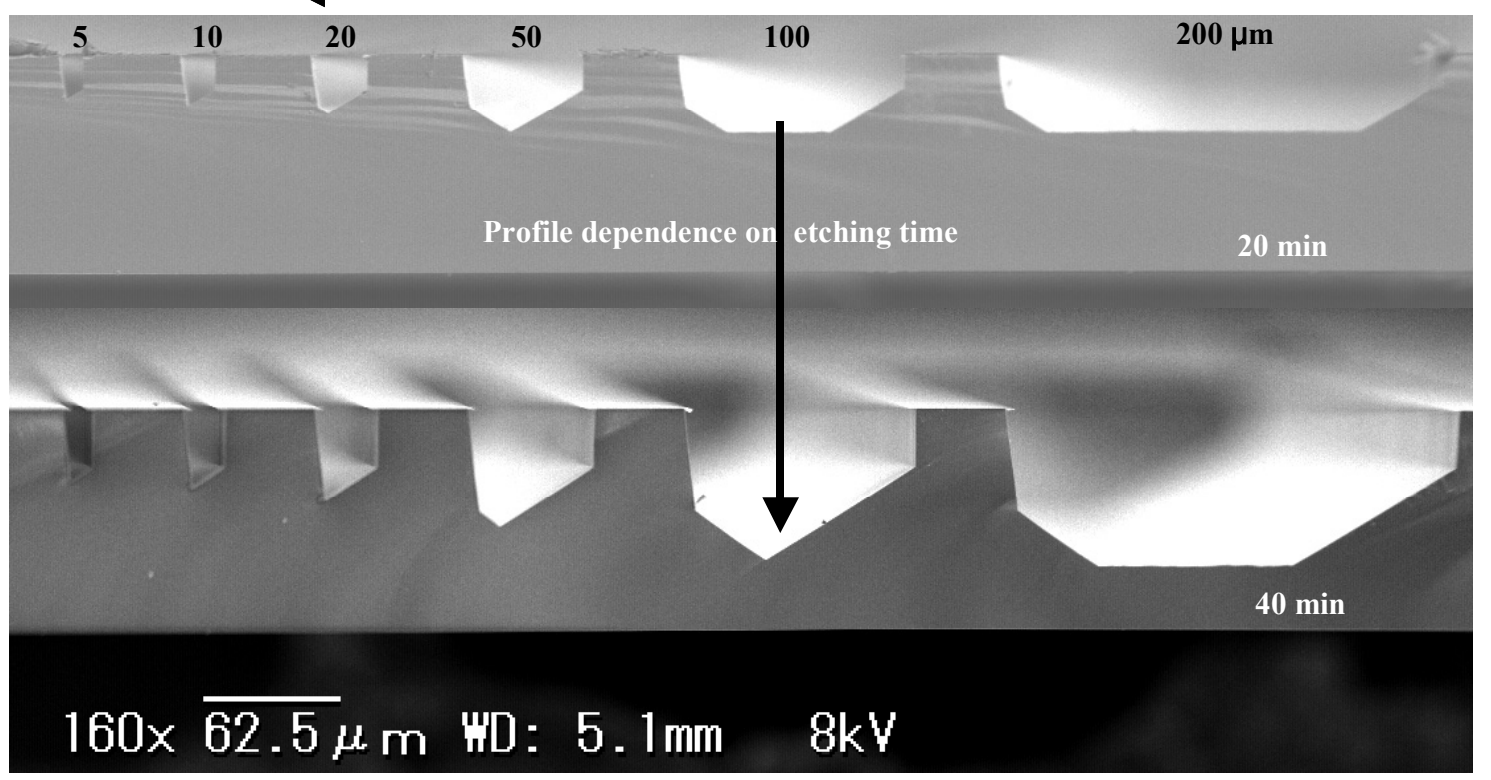

Fig. 6. SEM pictures of polar angle at $40^{\circ}$ for observing the trench profile dependence on initial width and etching time

and the intersection of $\mathrm{P}_{1}\left(\mathrm{P}_{2}\right), \mathrm{Au} / \mathrm{Cr}$ mask

\section{Results}

3.1 Characterization of the Anisotropy The angle $\alpha_{1}$, $\alpha_{2}, \alpha_{3}$ (in Fig. 2) were measured and plotted in dependence on the polar angle as Fig. 5(a). No minus inclination angle $\alpha$ was observed. We paid more attention on the angle $\alpha_{3}$, which tended to increase from $\beta=0^{\circ}$ to $15^{\circ}$ and from $\beta=90^{\circ}$ to $120^{\circ}$, but also suddenly decrease from $\beta=15^{\circ}$ to $30^{\circ}$. In a wide range from $\beta=30^{\circ}$ to $90^{\circ}$, it appeared to be nearly $0^{\circ}$. In fact, it was difficult to measure and discriminate the angle in this range, because no bigger than $2^{\circ}$ was observed. The side wall etching rates of $V_{1}, V_{2}$, $V_{3}$ were calculated and plotted as Fig. 5 (b). The etching rate $V_{Z}$ was $110 \mu \mathrm{m} / \mathrm{h}$, which was the same at all polar directions. So we did not plot $\mathrm{V}_{\mathrm{Z}}$.

3.2 Trench Profile Dependence on Initial Width and Etching Time Fig. 6 shows the typical SEM pictures including all initial widths $(5,10,20,50,100$ and $200 \mu \mathrm{m})$. These SEM pictures were used to study high aspect ratio microstructures.

3.3 High Aspect Ratio Microstructure For MEMS applications, high aspect ratio microstructures are required in microdevices design. Aspect ratio was defined as depth/width. Structures at polar angel $\beta$ should have a opposite shape with at polar angel $\beta+60^{\circ}$ because of the threefold symmetry property. So we just discussed the trench structures from $\beta=0^{\circ}$ to $60^{\circ} .40 \mathrm{~min}$ etched results are presented in Fig.7.

According to the $40 \mathrm{~min}$ etching results (Fig. 7), we defined the basic microstructure as it is presented in Fig. 8 (a). The $20 \mathrm{~min}, 40$ min and 70 min etched results with initial width $5 \mu \mathrm{m}$ were plotted in Fig. 8 (c). The aspect ratio did not arise as the increase of etching time due to the disappering of $\mathrm{Z}$ plane, $\mathrm{P}_{1}$ or $\mathrm{P}_{2}$ as shown in Fig. 6. Based on the 40 min results shown in Fig. 7, the trench profiles can be classified as (1) $\beta=0^{\circ}$ to $15^{\circ}$, in this range $Z$ plane and $\mathrm{P}_{1}$ disappeared rapidly and high aspect ratio structure can not be achieved because of the big angle $\alpha_{3}$ (Fig. 5 (a)); (2) $\beta=15^{\circ}$ to $30^{\circ}, \mathrm{P}_{2}$ plane appeared as bottom surface, but still no high aspect ratio structure can be achieved because of the low etching rate of $\mathrm{V}_{2}$ (Fig. 5 (b)); (3) $\beta=30^{\circ}$ to $45^{\circ}, \alpha_{2}$ increased gradually, but $\mathrm{V}_{2}$ increased quickly. So high aspect ratio microstructure can be achieved in this area; and (4) $\beta=45^{\circ}$ to $60^{\circ}, V_{2}$ became bigger, but attention should be paid to the side $B$, where $\alpha_{2}$ appeared gradually, and $\alpha_{3}$ became bigger. At $\beta=60^{\circ}$, feature was achieved and 


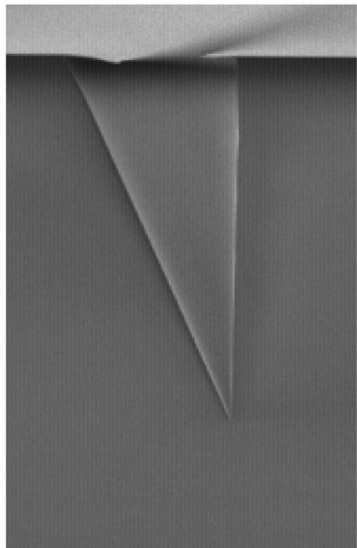

(1) $\beta=0^{\circ}$

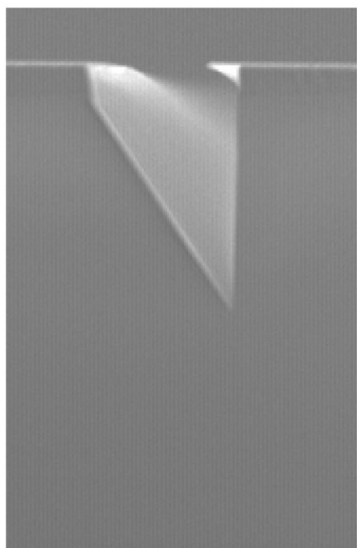

(5) $\beta=20^{\circ}$

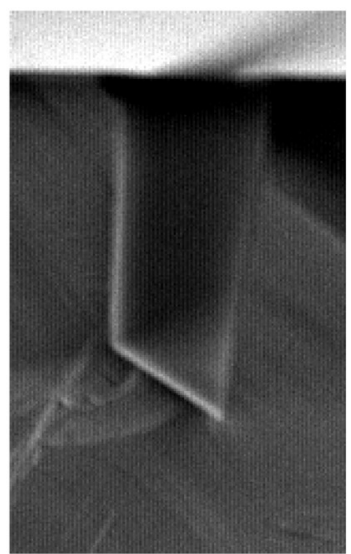

(9) $\beta=40^{\circ}$

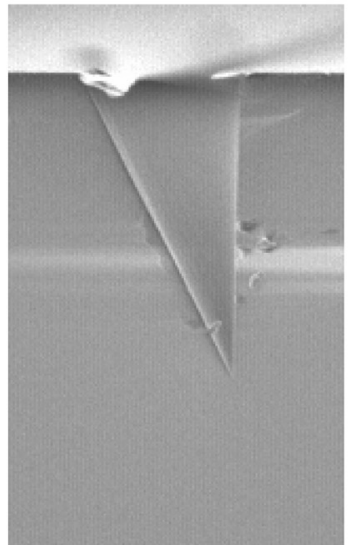

(2) $\beta=5^{\circ}$

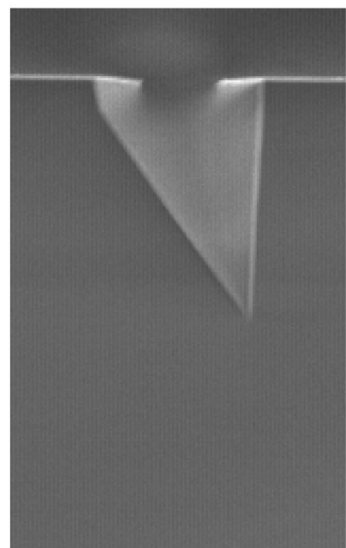

(6) $\beta=25^{\circ}$

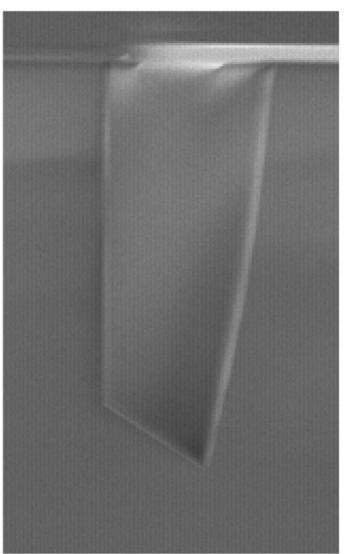

(10) $\beta=45$

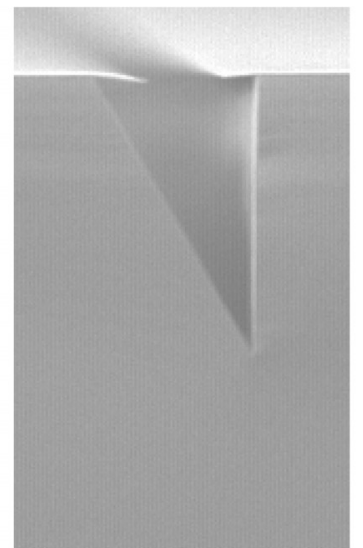

(3) $\beta=10^{\circ}$

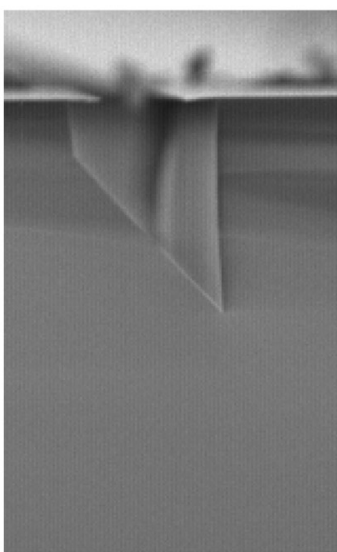

(7) $\beta=30^{\circ}$

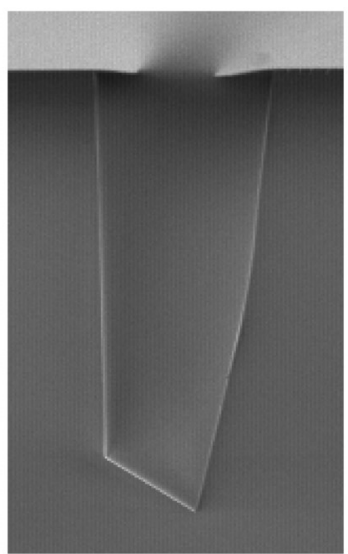

(11) $\beta=50^{\circ}$

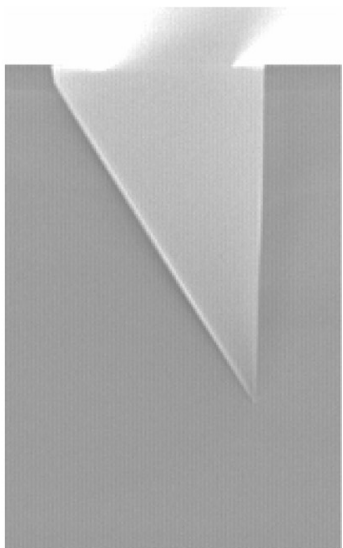

(4) $\beta=15^{\circ}$

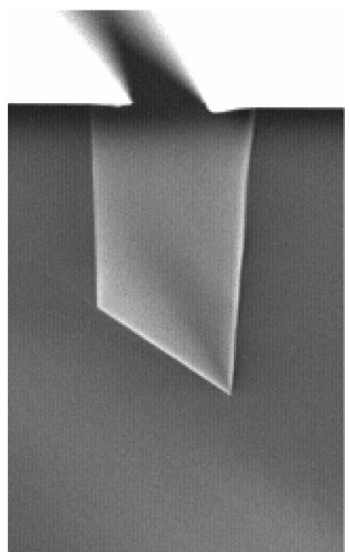

(8) $\beta=35^{\circ}$

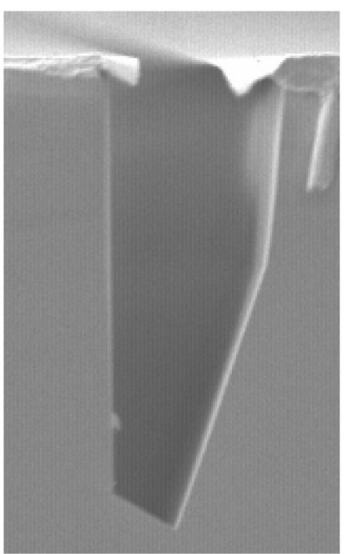

(12) $\beta=55^{\circ}$

$20 \mu \mathrm{m}$

Fig. 7. Cross section SEM pictures from $\beta=0^{\circ}$ to $55^{\circ}$ for 40 min etching with a initial width of $5 \mu \mathrm{m}$

returned to the $\beta=0^{\circ}$ feature. Fig. 8 (b) was an example SEM picture of 70 min etched trench with initial width $5 \mu \mathrm{m}$ at $\beta=45^{\circ}$.

\section{Discussion and Applications}

Double-sided etching experiment was also performed using the same mask pattern. The result showed that the bottom side has the same etching profile at the opposite polar direction $(\beta)$ due to the twofold symmetry in the positive $\mathrm{X}$ axis (Fig. 9).
In the case of

microchannel for micorcapillary, rectangular channel should be ideal, because slope side wall decreases the UV light pass length, resulting in low sensitivity. Considering the side etching $\left(\mathrm{u}_{1}, \mathrm{u}_{2}\right)$, side wall slope $\alpha$, and buttom surface etching rate $V_{2}, \beta=45^{\circ}$ should be the best choice. Fig. 8 (b) shows the 70 min etched trench with an aspect ratio of 3.1 at $\beta=45^{\circ}$.

4.2 Application for Capacitive Tilt Sensor For capacitance based sensor, quartz wafer should be etched through for achieving movable part and static part, so called 


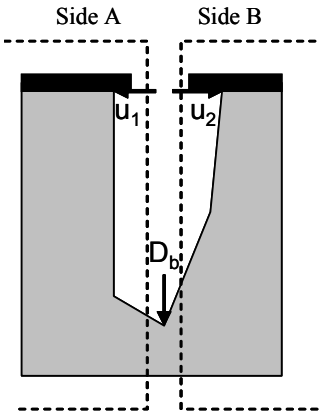

(a)

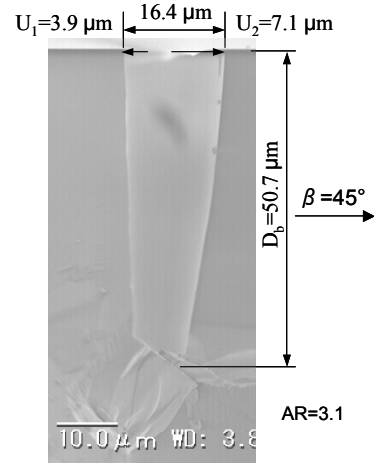

(b)

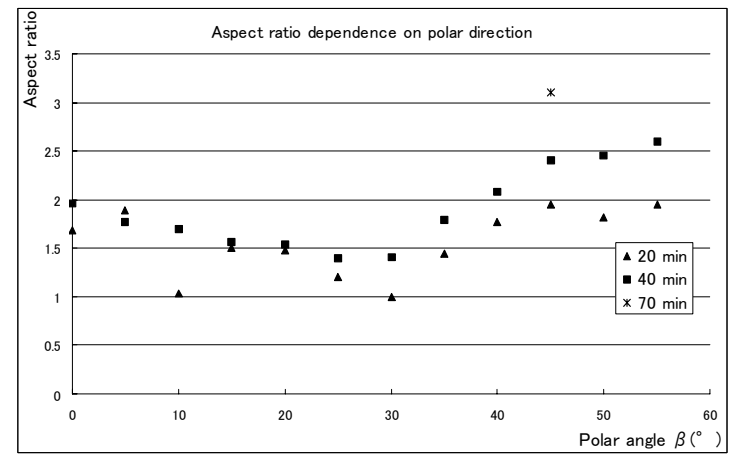

(c)

Fig. 8. Definition of the basic microstructure and aspect ratio: (a) the basic microstructure, $\mathrm{u}_{1}, \mathrm{u}_{2}$, and $\mathrm{D}_{\mathrm{b}}$ represent side etch and etch depth respectively; (b) SEM photograph of 70 min etched trench at $45^{\circ}$; (c) Plotted trench aspect ratio $\left[A R=D_{b} /\left(\right.\right.$ initial width $\left.\left.+u_{1}+u_{2}\right)\right]$ dependence on the polar direction based on the 20, 40, 70 min etching results

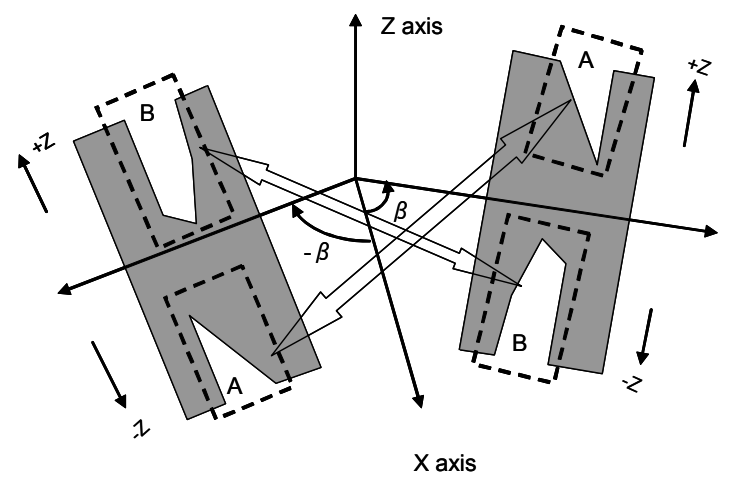

Fig. 9. The twofold symmetry around $X$ axis

comb-electrode. The key feature of this kind of sensor is to fabricate high aspect ratio through-hole, which is used for constructing variable capacitor. Double-sided etching technique shows great interest for the twofold symmetry. We have developed a tilt sensor ${ }^{(9)}$ using $+X$ direction $\left(\beta=0^{\circ}\right)$ for the high etching rate of $V_{1}$ (both sides of up and down) and simple side wall profile (up-down symmetry). The sensor is composed of comb-electrode, mass proof and long elastical cantilever. Fig. 9 shows the pictures of the fabricated tilt sensor.

In general consideration, for designing two axis sensor on one chip, sensor 2 will be arranged $120^{\circ}$ to the sensor 1 ( $+\mathrm{x}$ direction) for the well-known threefold symmetry property. However, $90^{\circ}$

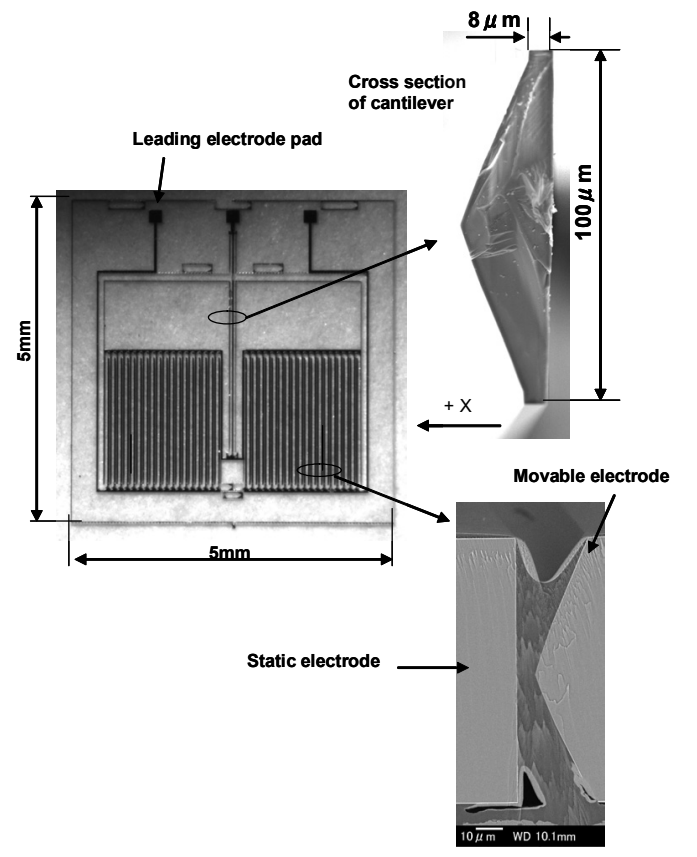

Fig. 10. Fabricated capacitive tilt sensor using $+\mathrm{X}$ direction

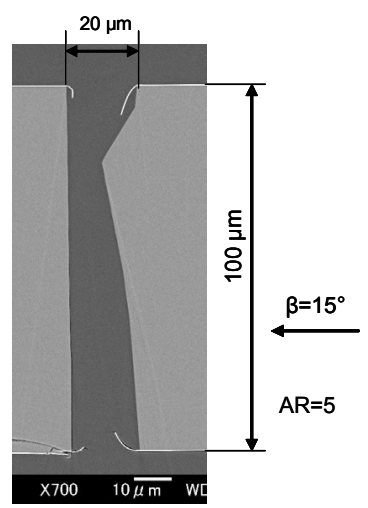

Fig. 11. Cross section of double-sided etched high aspect ratio $(\mathrm{AR}=5)$ through-hole at $\beta=15^{\circ}, 90 \mathrm{~min}$

arranged two axis sensor should also be achieved, if we let $\beta$ satisfy the following formula (6) taking advange of the twofold symmetry around $\mathrm{X}$ axis (Fig. 9).

$$
\beta-(-\beta)=90^{\circ}
$$

Namely, at $\beta=45^{\circ}$ and $-45^{\circ}$ same two sensors can be achieved, which just have up-down counter etching profiles. Further, considering the threefold symmetry property, two same sensors can also be achieved if satisfying the follwing formula (7), which gives $\beta=15^{\circ}$, arranging two sensors at $\beta=15^{\circ}$ and $105^{\circ}$ $\left(-15^{\circ}+120^{\circ}\right)$.

$$
120-\beta-\beta=90^{\circ}
$$

Actually, through-holes at $\beta=45^{\circ}$ and $105^{\circ}$ have the same etching profiles, which are only left-right counter. In conclusion, there is only one kind of through-hole etching profile which can be used for fabricating $90^{\circ}$ arrangement two axis tilt sensor. Fig. 11 shows the cross section of double-sided etched high aspect ratio through-hole at $\beta=15^{\circ}$, which was etched with an initial width $5 \mu \mathrm{m}$ for $90 \mathrm{~min}$. 


\section{Conclusion}

High aspect ratio microstructure $(>3)$ which was considered impossible by wet etching technology ${ }^{(3)}$ was achieved. This result suggests that anisotropic etching of $Z$ cut $\alpha$-quartz can be applied to microcapillary electrophoresis, where high aspect ratio microchannel is desired for improving detecting sensitivity. The dependence of aspect ratio on polar angle would give important guidance to the design layout. Trench at polar angle of $\beta=45^{\circ}$ is demonstrated to be the best choice. We successfully fabricated nearly vertical trench feature at $\beta=45^{\circ}$ with $50.7 \mu \mathrm{m}$ depth and $16.4 \mu \mathrm{m}$ width, the aspect ratio of 3.1. Double-sided etching showed great interest for the twofold symmetry around the $\mathrm{X}$ axis. According to this property, two same sensors at $\left(\beta=15^{\circ}\right.$ and $\left.105^{\circ}\right)$ and $\left(\beta=-45^{\circ}\right.$ and $\left.45^{\circ}\right)$ can be arranged at $90^{\circ}$ on one quartz chip, which propose a novel two axis tilt sensor.

\section{Acknowledgement}

This research has been supported by the Grant-In-Aid of Fukuoka Industry, Science and Technology Foundation (IST).

(Manuscript received Jul. 24, 2006, revised Jan. 4, 2007)

\section{References}

(1) T. Hattori : "Recent Advances and Prospects of precise 3D Microfabrication with a High Aspect Ratio", IEEJ Trans on SM, Vol.126, No.6, pp.211-215 (2006-6) (in Japanese)

(2) L. Ceriotti, K. Weible, N. F. de Rooij, and E. Verpoorte : "Rectangular Channels for Lab-on-a-chip applications", Microelectronic Engineering, Vol.67-68, pp.865-871 (2003)

(3) T. Ujiie, T. Kikuchi, T. Ichiki, and Y. Horiike : "Fabrication of Quartz Microcapillary Electrophoresis Chips Using Plasma Etching", Jpn. J. Appl. Phys., Vol.39, pp.3677-3682 (2000)

(4) Z. Liang, N. Chiem, G. Ocvirk, T. Tang, K. Fluri, and D. J. Harrison : "Microfabrication of a Planar Absorbance and Fluorescence Cell for Integrated Capillary Electrophoresis Devices", Anal. Chem, Vol.68, pp.1040-1046 (1996)

(5) T. Ueda, F. Kohsaka, T. Ino, and D. Yamazaki : "Theory to Predict Etching Shapes in Quartz Crystal and Its Applications to Design Devices". Trans. Soc. Instrum. Control Eng., Vol.23, No.12, pp.1233-1238 (1987, in Japanese)

(6) D. Cheng, K. Sato, M. Shikida, A. Ono, K. Sato, K. Asaumi, and Y. Iriye : "Characterization of Orientation-Dependent Etching Properties of Quartz: Application to 3-D Micromachining Simulation System". Sensors and Materials, Vol.17, No.4, pp.179-186, (2005)

(7) C. Hedlund, U. Lindberg, U. Bucht, and J. SÖderkvist : "Anisotropic etching of Z-cut quartz", J. Micromech. Microeng., Vol. 3, pp.65-73 (1993)

(8) T. Ueda : "Study on quartz microfabrication and its application to temperature sensor", pp.40 (Doctoral thesis, 1988, in Japanese)

(9) F. Kohsaka, J. Liang, and T. Ueda, "High Sensitive Tilt Sensor for Quartz Micromachining", Proceedings of the 22nd Sensor Symposium., pp,371-374, 2005 .
Jinxing Liang

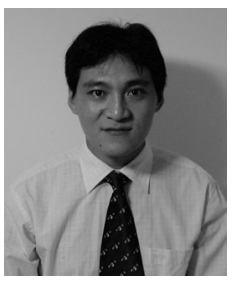

MEMS.

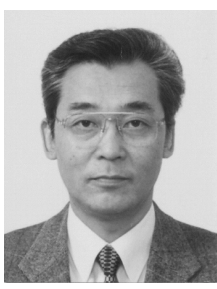

Takahiro Matsuo

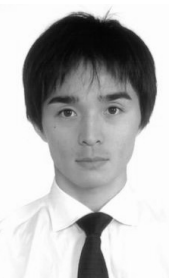

Fusao Kohsaka

(Member) Jinxing Liang was born on December 13, 1976. He received the B.E. degree in mechanical engineering from China University of Mining and Technology, China, in 1998. Then he received the M.E. degree in electrical engineering from Kyushu university, Japan, in March 2004. He joined Sakamoto Electric MFG. Co. Ltd, in April 2004, where he is currently working on quartz based

(Student Member) was born in 1952. He received the B.S. degree of mechanical engineering from Akita University in 1975. He joined Yokogawa Electric Corporation in 1975, where he engaged in research and development of sensors of displacement, pressure, temperature and rotational angle. Currently, he is developing Liquid Crystal Display, and now, he is a Ph. D. student in Waseda University.

(Student Member) He was born on May 26, 1976. He received his B.S. and M.S. degree in electrical engineering from Saga University in 1999 and 2001 respectively. He has worked in Rohm Apollo Device Co., LTD from 2001 to 2004 . He is currently working on Ph.D degree at Graduate School of Information, Production and Systems in Waseda University while being an employee of Sakamoto

Electric MFG.Co., Ltd.

Toshitsugu Ueda (Member) Toshitsugu Ueda was born in Nara, Japan,

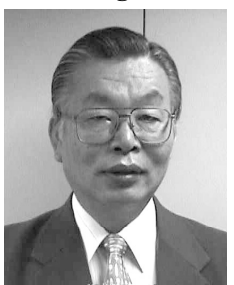
on October 4, 1945. He received the B.E. and M.E. degree in electrical engineering from Shinshu University, Nagano, Japan, in 1969 and 1971 respectively. He received Ph.D. degree from Tokyo Institute of Technology in 1988. Since joining Yokogawa Electric Corporation in 1971, he has been engaged in developing low noise amplifiers, mechanical resonators, micro machining technologies and sensors using above mentioned technologies for temperature, pressure and displacement. Now he is a professor of Waseda University. He received Awards from Society of Instrument and Control Engineers of Japan in 1987 and 1994, and Awards from Japan Institute Invention and Innovation in 1985 and 1987. Dr. Ueda is a member of the Institute of Electrical Engineers of Japan, and Society of Instrument and Control Engineers of Japan. 